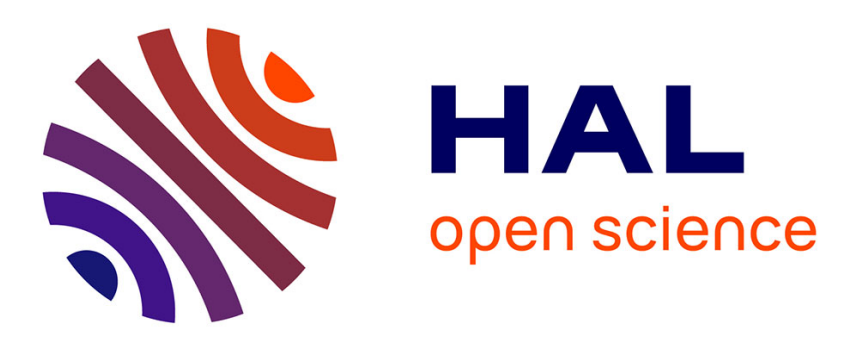

\title{
Doubly-resonant SFG and DFG spectroscopies: an analytic model for data analysis including distorted and rotated vibronic levels. II. Applications \\ Bertrand Busson
}

\section{- To cite this version:}

Bertrand Busson. Doubly-resonant SFG and DFG spectroscopies: an analytic model for data analysis including distorted and rotated vibronic levels. II. Applications. Journal of Chemical Physics, 2020, 153 (17), pp.174702. 10.1063/5.0022761 . hal-02965974

\author{
HAL Id: hal-02965974 \\ https://hal.science/hal-02965974
}

Submitted on 13 Oct 2020

HAL is a multi-disciplinary open access archive for the deposit and dissemination of scientific research documents, whether they are published or not. The documents may come from teaching and research institutions in France or abroad, or from public or private research centers.
L'archive ouverte pluridisciplinaire HAL, est destinée au dépôt et à la diffusion de documents scientifiques de niveau recherche, publiés ou non, émanant des établissements d'enseignement et de recherche français ou étrangers, des laboratoires publics ou privés. 
Doubly-resonant SFG and DFG spectroscopies: an analytic model for data analysis including distorted and rotated vibronic levels. II. Applications

\author{
Bertrand Busson ${ }^{1}$ \\ Université Paris-Saclay, CNRS, Institut de Chimie Physique, UMR 8000, \\ 91405 ORSAY, France ${ }^{\mathrm{a})}$
}

(Dated: 28 September 2020)

The influence of vibration mode distortion and mode mixing in the vibronic structure of molecules on the doubly resonant sum and difference frequency generation spectroscopies is systematically studied on several examples. These phenomena modify the spectral overlap function at the heart of the modeling of the doubly resonant processes. When the visible beam is tuned, under the Franck-Condon approximation, each vibration mode generates two main peaks corresponding to the resonance of the molecular electronic transition with the visible and SFG energies, together with higher order ones driven by the amplitudes of the vibronic displacements. For a single mode system, mode distortion modifies the positions of the main peaks and the excitations of the higher order ones. For a two-mode system, mode mixing induces in addition a deep change in the balance between the intensities of the mixed modes. It also changes the phases of the vibrations, which makes curve fitting difficult. For multimode systems, mode mixing may greatly enhance intensity of a poorly active mode. For all these reasons, it seems mandatory to take mode distortion and mode mixing into account for an accurate analysis of second-order nonlinear spectroscopic experimental data.

\footnotetext{
${ }^{a)}$ Electronic mail: bertrand.busson@universite-paris-saclay.fr
} 


\section{INTRODUCTION}

In a previous paper, ${ }^{1}$ we have established the formulas for the account of mode distortions and mode mixing in the excitation spectra in doubly resonant infrared-visible Sum and Difference Frequency Generation spectroscopies. Here we apply these results on several test cases with growing complexity in order to evaluate their implication on the analysis of experimental data.

In a doubly resonant infrared-visible SFG process, the infrared (IR) beam (frequency $\omega_{I R}$ ) resonantly induces a vibrational transition from the ground state and excites a vibration mode $(l)$. The visible and SFG beams may become resonant with vibronic transitions and, in the Condon approximation, all totally symmetric vibrations are Franck-Condon allowed. In the usual linear electron-vibration coupling (LC superscripts in the following) scheme ${ }^{2}$ for which all vibration modes $\{m\}$ in the excited state exactly match those in the ground state $\left(\{j\}\right.$, energy $\hbar \omega_{j}$, width $\left.\Gamma_{j}\right)$ except for a displacement $\Delta_{j}$ along the vibration coordinate, the doubly resonant SFG molecular hyperpolarizability may be written as:

$$
\beta_{i j k}^{S F G}=1 / \hbar^{2} \sum_{l}^{I R-a c t i v e} \frac{A_{l}^{i j k} D_{l}^{L C}\left(\omega_{S F G}\right)}{\omega_{I R}-\omega_{l}+i \Gamma_{l}}
$$

where $A_{l}^{i j k}=\mu_{g \leftarrow e}^{0, i} \mu_{e \leftarrow g}^{0, j}\left(\frac{\partial \mu^{k}}{\partial Q_{l}}\right)_{Q_{l}=0}$ is the static SFG amplitude and involves the dipole moment of the electronic and vibrational transitions; $D_{l}^{L C}(\omega)$ is the excitation spectrum of mode $l$. We have

$$
D_{l}^{L C}(\omega)=\frac{\Delta_{l}}{2}\left[\Phi^{L C}(\omega)-\Phi^{L C}\left(\omega-\omega_{l}^{e}\right)\right]
$$

where we introduce the overlap spectral function in the linear coupling scheme $\Phi^{L C}(\omega)$. This function may be evaluated either experimentally, for example from an absorption spectrum $A(\omega)$ as $A(\omega) \propto \omega\left|\mu_{e \leftarrow g}^{0}\right|^{2} \operatorname{Im}[\Phi(\omega)]$, or by a direct calculation. ${ }^{1}$

When quadratic electron-vibration couplings are taken into account, the vibronic structure does not match the ground state vibration modes as vibronic modes may be distorted (hence frequency-shifted) and mixed (through Duschinsky rotation). In this case, it is necessary to differentiate vibration frequencies $\omega_{j}^{g}$ and $\omega_{j}^{e}\left(\right.$ with $\left.\lambda_{j}=\omega_{j}^{e} / \omega_{j}^{g}\right)$ in the ground and excited states, respectively. When only mode distortions (DIS superscripts) occur, the SFG molecular hyperpolarizability becomes:

$$
\beta_{i j k}^{S F G}=1 / \hbar^{2} \sum_{l}^{I R-\text { active }} \frac{A_{l}^{i j k} D_{l}^{D I S}\left(\omega_{S F G}\right)}{\omega_{I R}-\omega_{l}^{g}+i \Gamma_{l}^{g}}
$$


with, for low distortions (i.e. $\lambda_{j}$ close to 1 ):

$$
\begin{gathered}
D_{l}^{D I S}(\omega)=\frac{\Delta_{l}}{4}\left(\left(1+\lambda_{l}\right) \Phi^{F S}(\omega)-2 \Phi^{F S}\left(\omega-\omega_{l}^{e}\right)+\left(1-\lambda_{l}\right) \Phi^{F S}\left(\omega-2 \omega_{l}^{e}\right)\right. \\
\left.+\sum_{j=1}^{N} S_{j}\left(1-\lambda_{j}\right)\left[\Phi^{F S}(\omega)-\Phi^{F S}\left(\omega-\omega_{l}^{e}\right)-\Phi^{F S}\left(\omega-2 \omega_{j}^{e}\right)+\Phi^{F S}\left(\omega-\omega_{l}^{e}-2 \omega_{j}^{e}\right)\right]\right)
\end{gathered}
$$

where function $\Phi^{F S}(\omega)$ is formally identical to $\Phi^{L C}(\omega)$ but now takes vibronic frequency shifts into account (hence the FS superscripts):

$$
\Phi^{F S}(\omega)=e^{-S} \sum_{\substack{k_{1}=0 \\(\ldots) \\ k_{N}=0}}^{\infty}\left(\prod_{j=1}^{N} \frac{\left(S_{j}\right)^{k_{j}}}{k_{j} !}\right) \frac{(-1)}{\omega-\omega_{e g}^{0}-\sum_{j=1}^{N} k_{j} \omega_{j}^{e}+i \Gamma_{e g}}
$$

where $S=\sum_{j=1}^{N} S_{j}$ with $S_{j}=\frac{\omega_{j}^{g}\left(\Delta_{j}\right)^{2}}{2 \hbar}=\frac{\left(\bar{\Delta}_{j}\right)^{2}}{2} ; \omega_{e g}^{0}$ and $\Gamma_{e g}$ define the vibrationless energy and width (supposed constant in the whole vibronic structure) of the electronic transition, respectively. Summation over j runs over the $\mathrm{N}$ modes of the vibronic structure, and index $k_{j}$ indicates the order of excitation of mode $\mathrm{j}$.

When two modes (numbered 1 and 2) are involved in mode mixing (MIX superscripts) with rotation angle $\theta$, supposed small, the SFG molecular hyperpolarizability is further modified by addition of $\theta$-dependent terms:

$$
\beta_{i j k}^{S F G}=\frac{1}{\hbar^{2}}\left(\sum_{l}^{I R-\text { active }} \frac{A_{l}^{i j k} D_{l}^{M I X}\left(\omega_{S F G}\right)}{\omega_{I R}-\omega_{l}^{g}+i \Gamma_{l}^{g}}+\frac{A_{1}^{i j k} D_{1}^{C T}\left(\omega_{S F G}\right)}{\omega_{I R}-\omega_{1}^{g}+i \Gamma_{1}^{g}}+\frac{A_{2}^{i j k} D_{2}^{C T}\left(\omega_{S F G}\right)}{\omega_{I R}-\omega_{2}^{g}+i \Gamma_{2}^{g}}\right)
$$

where functions $D_{l}^{M I X}(\omega)=D_{l}^{D I S}(\omega)+D_{l}^{\theta}(\omega)$ and complementary terms (CT superscripts) $D_{1,2}^{C T}(\omega)$ are recalled in the Appendix. As mode mixing is a perturbation of the distorted case, Eq. 6 may be generalized to an arbitrary number of modes mixed by pairs through the addition of several new terms per pair. ${ }^{1}$ The total hyperpolarizability becomes in this case:

$$
\beta_{i j k}^{S F G}=\frac{1}{\hbar^{2}} \sum_{l}^{I R-\text { active }} \frac{A_{l}^{i j k}}{\omega_{I R}-\omega_{l}^{g}+i \Gamma_{l}^{g}}\left[D_{l}^{D I S}\left(\omega_{S F G}\right)+D_{l}^{\theta}\left(\omega_{S F G}\right)+D_{l}^{C T}\left(\omega_{S F G}\right)\right]
$$

where $D_{l}^{D I S}(\omega)$ is still given by Eq. $4, D_{l}^{\theta}(\omega)$ now sums up the contributions from all pairs of mixed modes (Eq. A5) while $D_{l}^{C T}(\omega)$ is shown in the Appendix when mode $l$ is involved in mode mixing, and vanishes otherwise. As a consequence of Eq. 7, all the hyperpolarizability components $\beta_{i j k}$ share a common dispersion in the visible for a fixed $\omega_{I R}$, determined by $D_{l}$. It is therefore also the case for the nonlinear susceptibility $\chi^{(2)}$ components and, provided 
that the Fresnel factors don't vary in the visible range, for the effective surface susceptibility. ${ }^{3}$ Consequently, the SFG dispersion in the visible range, as calculated here at the molecular level for $\beta_{i j k}$, should be the same for experimental spectra recorded while tuning the input visible frequency.

\section{APPLICATIONS}

We now illustrate the theoretical results above in three steps, following the structure of Paper I. ${ }^{1}$ Firstly, we propose an efficient way for the evaluation of function $\Phi^{F S}(\omega)$ as a preliminary to the calculation of excitation spectra (part II A). We then rely on the simple cases of one-mode and two-mode systems to understand the effects of distortion (part II B) and mode mixing (part II C), respectively, on the excitation and SFG spectra. Finally, we consider more realistic molecular models and illustrate the interference effects (part IID) and energy transfers (part II E) between vibration modes.

\section{A. Calculation of the overlap spectral function}

The $\Phi^{F S}(\omega)$ formalism allows to simplify the actual evaluation of the vibronic contribution. As the heart of the transform method, it may be deduced from the absorption spectrum and computed without a deep analysis. As mentioned above, in the case of FG, the adsorption of one monolayer or less on a substrate may dramatically change the absorption properties and make its accurate experimental measurement difficult. As for a direct calculation of $\Phi^{F S}$, it has to be performed only once and applies to all vibration modes. After this step, simulating DR-SFG/DFG spectra while playing with distortion and mode mixing parameters is rather straightforward, provided that the correct values of parameters $\lambda_{j}$ are used.

Care must be taken when calculating a numerical approximation for $\Phi^{F S}(\omega)$ based on Eq. 5. For example, a straight summation using nested loops over $k_{j}$ up to order $\mathrm{R}$ for $\mathrm{N}$ vibration modes implies to evaluate $(\mathrm{R}+1)^{\mathrm{N}}$ terms with very different weights. It is therefore necessary to optimize the calculation method by evaluating the terms with decreasing amplitude and choosing a cutoff for stopping the summation (i.e. for convergence). For an efficient method, we consider a truncated summation up to a total order $R=\sum_{j=1}^{N} k_{j}$, 
which comes to rewriting Eq. 5 under the form:

$$
\Phi^{F S}(\omega)=e^{-S} \sum_{R=0}^{\infty} \sum_{\substack{\left\{k_{j}\right\}_{1 \leq j \leq N} \\ \sum_{j=1}^{N} k_{j}=R}}\left(\prod_{j=1}^{N} \frac{\left(S_{j}\right)^{k_{j}}}{k_{j} !}\right) \frac{(-1)}{\omega-\omega_{e g}^{0}-\sum_{j=1}^{N} k_{j} \omega_{j}^{e}+i \Gamma_{e g}}
$$

The number of terms at a given total order $\mathrm{R}$ is equal to the number of R-permutations of $\mathrm{N}$ with repetition, that is $\left(\begin{array}{c}N+R-1 \\ R\end{array}\right)$. The number of terms in the sum with total orders ranging from 1 to $\mathrm{R}$ is $\left(\begin{array}{c}N+R \\ R\end{array}\right)$. Considering that the fractions of each term in the sum all weigh at most $1 / \Gamma_{e g}$, we focus on the coefficients in order to define a cutoff for convergence. Defining the weight $w(R)$ of a given total order $\mathrm{R}$ by the sum of the weights of all its terms, we have

$$
w(R)=\sum_{\substack{\left\{k_{j}\right\}_{1 \leq j \leq N} \\ \sum_{j=1}^{N} k_{j}=R}}\left(\prod_{j=1}^{N} \frac{\left(S_{j}\right)^{k_{j}}}{k_{j} !}\right)
$$

This quantity is equal to the R-term in the Taylor expansion of $e^{S}$ with $S=\sum_{j=1}^{N} S_{j}$, that is:

$$
w(R)=\frac{\left[\sum_{j=1}^{N} S_{j}\right]^{R}}{R !}=\frac{S^{R}}{R !}
$$

To choose a convergence cutoff, one may therefore define a threshold for $w(R)$, below which the higher orders are neglected and the summation truncated with essentially no consequence on the result. This is an alternate way to define classes of excitation accessible at a given level of coupling. ${ }^{4}$ For practical applications, we suggest to take as a reference the weight of order 1 for the definition of the threshold $T_{w}$. The condition for stopping the calculation before order $\mathrm{R}$ is known in advance and becomes:

$$
\frac{w(R)}{w(1)}=\frac{S^{R-1}}{R !}<T_{w}
$$

For small values of coefficients $S_{j}$, this happens very fast. With $T_{w}=1 \%$, the calculation stops after order 3 for $S=0.5$ (leading to only 23426 terms for 50 vibration modes) but goes up to order 7 for $S=2.0\left(2.3 \times 10^{8}\right.$ terms for 50 modes $)$. As an example, the $\Phi^{F S}(\omega)$ function built from the calculated vibronic structure of rhodamine $6 \mathrm{G}^{5}$ (R6G, 31 vibration modes, $\omega_{e g}^{0}=18800 \mathrm{~cm}^{-1} ; \Gamma_{e g}=400 \mathrm{~cm}^{-1}, S=0.38$, predicted order to stop calculation $=4$ ) has been 
calculated (Figure S1). The vibronic structure of this dye has been studied a lot, either by resonant Raman spectroscopy or first principle calculations. ${ }^{6-12}$ The maximum differences between the functions truncated at total orders 3 and 4 were below $0.5 \%$ in modulus and $0.1^{\circ}$ in phase. As can be seen in the Supplementary Material, the difference between orders 2 and 3 is already hardly detectable by eye. If we double the displacements $\Delta_{j}$, then $S$ goes up to 1.53 and the estimated maximal order becomes 7 at $1 \%$ convergence threshold. After calculation (Fig. 1), maximal difference in magnitude is $0.2 \%$ between orders 6 and 7 and $0.8 \%$ between orders 5 and 6 . The differences preferentially lie in the imaginary part, for which it becomes at most $1 \%$ between orders 6 and 7 as expected, and $3 \%$ between orders 5 and 6.

We note here the differences between real part, imaginary part and modulus. For absorption spectroscopy, only the imaginary part plays a role, leading to well-defined and narrow
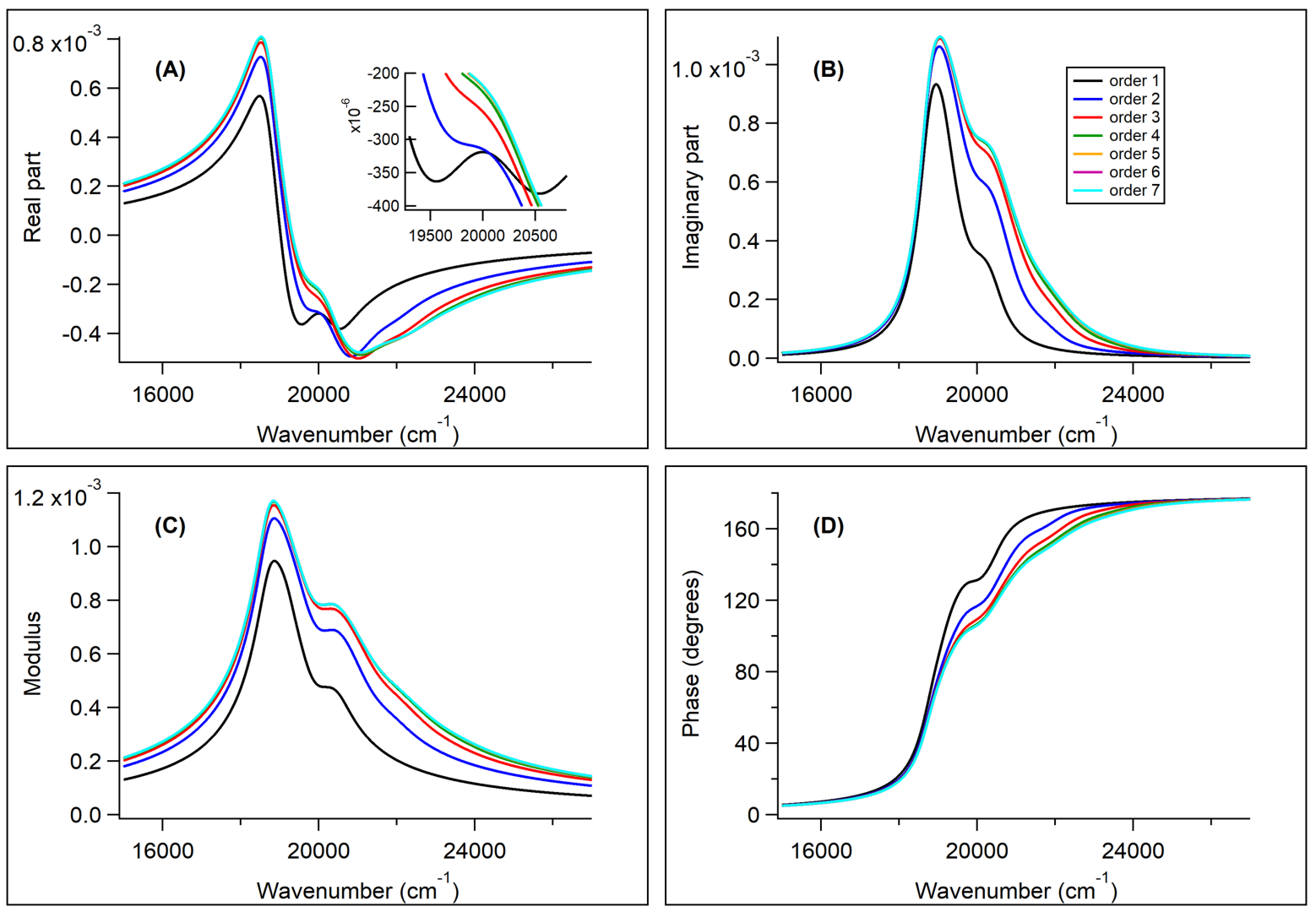

FIG. 1. Complex $\Phi$ function calculated for rhodamine $6 \mathrm{G}$ with 31 vibration modes using the parameters of Ref. 5 with all displacement parameters doubled. (A): real part; (B) imaginary part; (C) modulus; (D) complex phase. 
peaks pointing up above a zero background. On the contrary, in RRS and DR-SFG spectroscopies, as the modulus appears in the equations, the influence of the derivative shape of the real part modifies the properties of the resonances. The main consequences are a broadening of the resonance peak leading to nonvanishing contributions extending far from the resonance, with consequences on the intensity ratio measured in and out of resonance. This is a general phenomenon already noted when comparing absorption and SFG spectroscopies enhanced by coupling to either excitons ${ }^{13}$ or surface plasmons. ${ }^{14,15}$

\section{B. One-mode system: effects of mode distortions}

We analyze the effects of mode distortion alone in the model case of one vibration mode. In the following, we label first, second, etc... all the peaks appearing on the curves or 3D plots in the increasing energy order, including the 0-0 transition. For this example, we choose the parameters from one of the most intense bands in the calculated RRS spectra of rhodamine

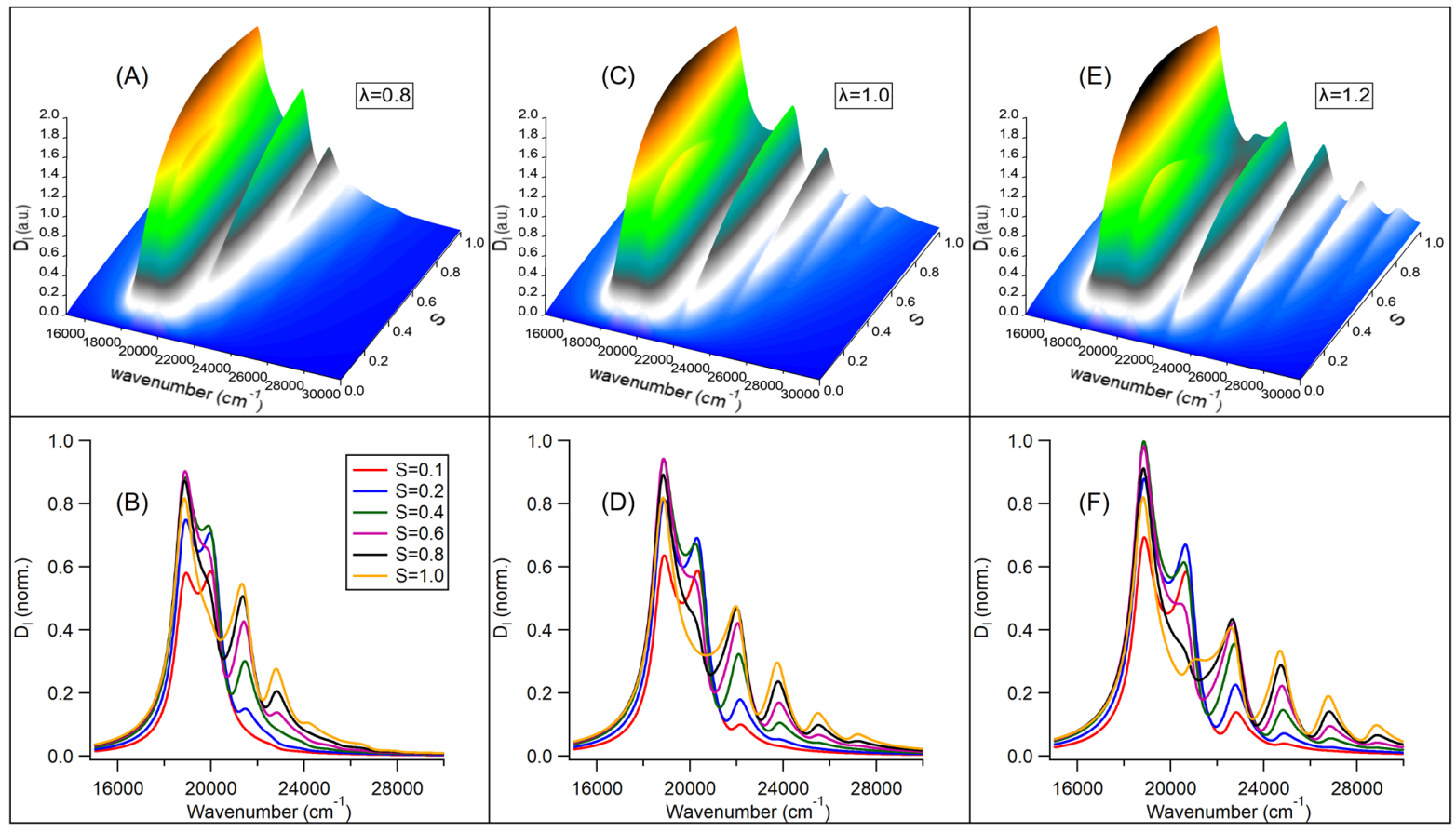

FIG. 2. Amplitude of function $D_{l}$ in a distorted one-mode system as a function of wavenumber $\omega$ and Huang-Rhys factor $\mathrm{S}$ for three values of distortion parameter $\lambda$ : $0.8(\mathrm{~A}, \mathrm{~B}) ; 1.0(\mathrm{C}, \mathrm{D})$ and $1.2(\mathrm{E}, \mathrm{F})$. See text for parameter values. Top row: absolute amplitudes. Bottom row: amplitudes normalized to the highest value of main peak at $\lambda=1.2$. 
$6 \mathrm{G}^{5}: \omega_{e g}^{0}=18800 \mathrm{~cm}^{-1} ; \Gamma_{e g}=400 \mathrm{~cm}^{-1} ; \omega^{g}=1652 \mathrm{~cm}^{-1}$. The two variable parameters are the Huang-Rhys factor S, tuned from 0 to 1.0, and the distortion factor $\lambda$, between 0.7 and 1.3. Such intervals allow to illustrate the behaviour of the excitation function. For realistic systems, $\lambda$ usually lies between 0.9 and $1.1, \bar{\Delta}$ below 0.4 , hence $\mathrm{S}$ below 0.1 . The variation in magnitude of the excitation profile $D(\omega)$ as a function of $\mathrm{S}$ and the wavenumber has already been studied, ${ }^{2}$ and we extend it in Fig. 2 to several mode distortion parameters $\lambda$ (additional curves may be found in the Supplementary Material). In the 3D plots, absolute values are displayed and we extract some $2 \mathrm{D}$ profiles for several values of $\mathrm{S}(0.1,0.2,0.4$, 0.6, 0.8 and 1.0). For the latter, we normalize all curves to the highest peak value in order to focus on the evolution of the lineshapes as a function of the visible wavenumber and distortion parameter $\lambda$. Parameter $\mathrm{S}$ drives the influence of higher order vibronic states in the excitation profile, whereas $\lambda$ shifts the positions and modulates the amplitudes of the higher order peaks, leading to a blueshift of the extremal peak in the excitation profile. The amplitude of the first and main peak (its position corresponds to $\omega_{e g}^{0}$ and does not depend on the parameters of vibronic structure) only weakly depends on $S$ (except for $S \leq 0.1$ ) because it varies approximately as $\sqrt{S} e^{-S}$, a rather smooth function between 0.1 and 1.4 , with a maximum at $S=0.5$. As already noticed, ${ }^{2}$ the amplitude of the second peak decreases for increasing $S$ as a consequence of interferences between resonances at the visible and SFG wavenumbers. For values close to $(S=1.0 ; \lambda=1.0)$, the peak disappears from the excitation profiles (Fig. 2D and 3F). On the contrary, for low $S$, the first two peaks have equivalent heights, with an overall small amplitude.

The influence of $\lambda$ values is more easily understood in Fig. 3, which displays the excitation profiles as a function of the wavenumber and the distortion parameter. As above, the 3D plots show absolute values, and the 2D extracts are normalized to the highest values. We see that $\lambda$ influences indeed the positions of the higher order peaks and has little influence on the amplitudes of the first ones. In particular, the height of the first peak remains almost constant with $\lambda$. On the contrary, the $\lambda$-dependent terms in Eq. 4 allow reaching higher order terms with lower powers of $S$, their amplitudes are therefore greatly influenced by $\lambda$. As expected from the development of Eq. 4, the third peak disappears for the particular coupled values $2 S=1-\lambda$ (e.g. $S=0.1$ and $\lambda=0.8$, Fig. $3 \mathrm{~B})$, in the same way as the second peak for $(S=1.0 ; \lambda=1.0)$.

In a first approximation, $S$ drives the overall amplitudes and the number of modes in the 


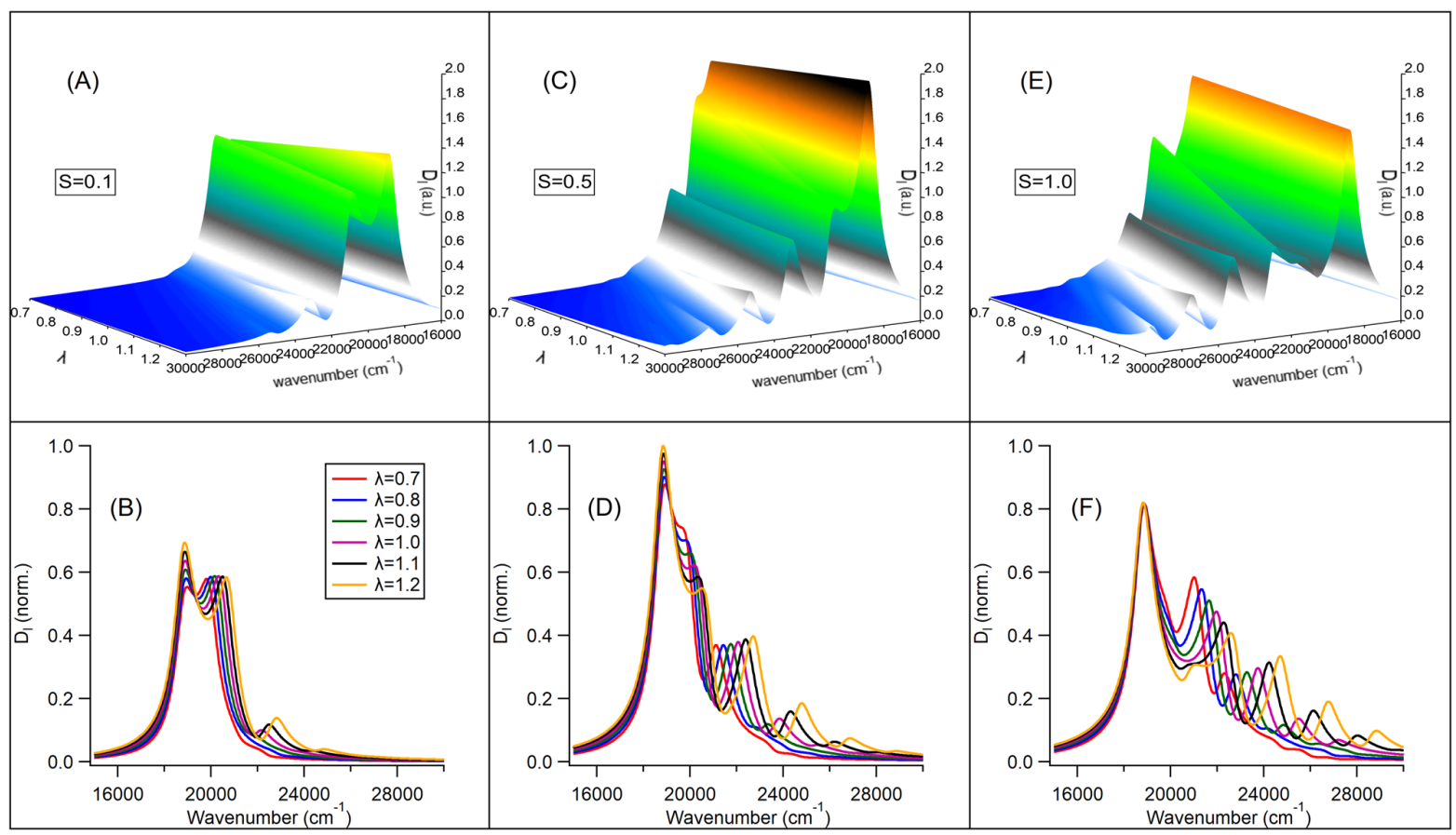

FIG. 3. Amplitude of function $D_{l}$ in a distorted one-mode system as a function of wavenumber $\omega$ and distortion parameter $\lambda$ for three values of Huang-Rhys factor S: 0.1 (A,B); $0.5(\mathrm{C}, \mathrm{D})$ and $1.0(\mathrm{E}, \mathrm{F})$. See text for parameter values. Top row: absolute amplitudes. Bottom row: amplitudes normalized to the highest value of main peak at $\mathrm{S}=0.5$.

excitation profiles towards high energies, whereas the distortion parameter $\lambda$ determines the positions of the higher order peaks, hence the extent of the excitation profile towards higher energies for a given value of S. In order to check this, we have selected in Fig. S5 the $\lambda$ values relevant for most realistic systems, and shown the influence of parameter $\lambda$ by comparing the excitation profiles calculated using the exact formula (Eq. 4) and approximated under the frequency-shifted linear coupling scheme (Eq. 4 with $\lambda=1$ ). We see that there is little difference in this $\lambda$ range and that it is possible, to a fair approximation, to limit the calculation of the influence of mode distortions to the induced frequency shifts in the vibronic structure. In other words, mode distortion has little impact on the excitation profiles except for the positions in energy of the vibronic resonances.

The actual excitation profile for a given mode corresponds to $D_{l}(\omega)$ evaluated at the SFG frequency (Eq. 3), thus the positions of the peaks will also depend on the frequency of the selected normal mode. In order to illustrate this, we plot on Fig. 4 the amplitudes of the excitation spectra (A-C: absolute values; D-F: normalized to 1) for several vibration mode 


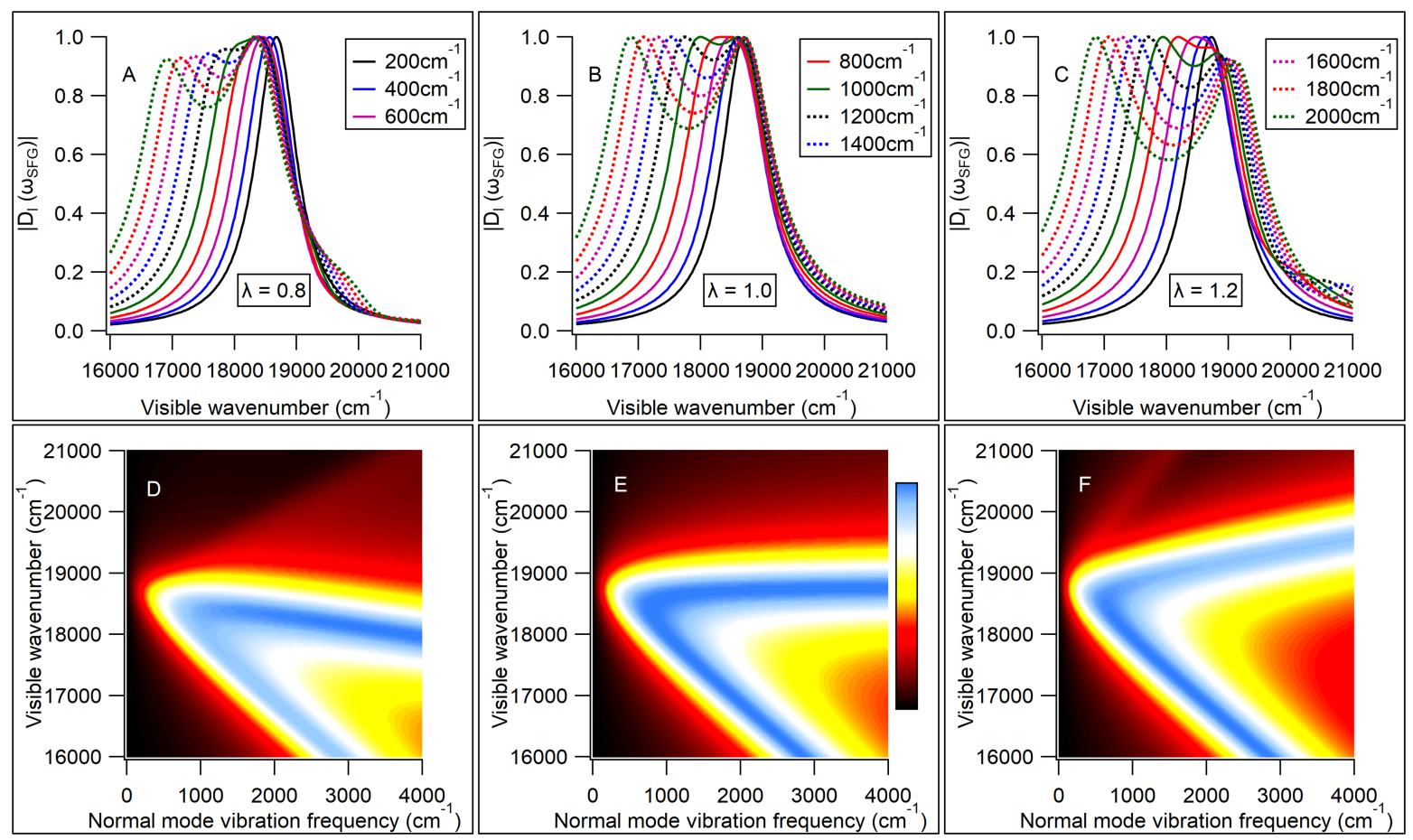

FIG. 4. Amplitude of function $D_{l}\left(\omega_{S F G}\right)$ in a distorted one-mode system as a function of incoming visible wavenumber and eigenfrequency of vibration mode $\omega_{l}^{g}$ (from 200 to $2000 \mathrm{~cm}^{-1}$ ), for three values of distortion parameter $\lambda(0.8 ; 1.0 ; 1.2)$ and Huang-Rhys factor $S=0.02$. A-C: $1 \mathrm{D}$ profiles normalized to 1. D-F: 2D maps with absolute values.

frequencies (considered alone in a one-mode system) for three values of distortion $\lambda$ at low $\mathrm{S}$ values. For high energy vibrations, the two excitation peaks are clearly seen, only the left one being independent of distortion (resonance at $\omega_{S F G}=\omega_{e g}^{0}$ ) and the right one shifting as expected to the blue with increasing $\lambda$. On the contrary, for low energy vibrations, the two peaks merge into one central peak, leading to a global broadening and redshift from the theoretical $\omega_{e g}^{0}$ position. Both peaks become distinguishable for $\omega_{l}^{e}$ bigger than $2 \Gamma_{e g}$. For broader electronic resonances, or for samples where inhomogeneous broadening becomes important, we may expect this single peak behaviour even for high energy modes. ${ }^{6}$. Even in the undistorted case (Fig. 4B), the right peak is always redshifted with respect to the theoretical $\omega_{v i s}=\omega_{e g}^{0}$ condition because of interference with the left peak. 


\section{Two-mode system: effects of mode mixing}

In order to illustrate the effects of mode mixing, we focus on a model two-mode system. The only published results on DR-SFG with mode mixing ${ }^{16}$ use the following fixed parameters: $\omega_{e g}=20000 \mathrm{~cm}^{-1} ; \Gamma_{e g}=90 \mathrm{~cm}^{-1} ; \omega_{1}^{g}=500 \mathrm{~cm}^{-1} ; \omega_{1}^{e}=520 \mathrm{~cm}^{-1}$; $\omega_{2}^{g}=800 \mathrm{~cm}^{-1} ; \omega_{2}^{e}=790 \mathrm{~cm}^{-1} ; \Gamma_{1}^{g}=\Gamma_{2}^{g}=20 \mathrm{~cm}^{-1}$ and equal IR mode activities, which means $A_{2}^{i j k} / A_{1}^{i j k}=\sqrt{\omega_{2}^{g} / \omega_{1}^{g}}$. The tunable parameters are therefore the mixing angle $\theta$ (standard value $-15^{\circ}$ ), the visible frequency $\omega_{v i s}$ (standard value $20000 \mathrm{~cm}^{-1}$ ) and the vibrational displacements. The authors have chosen to control the vibrational displacements through parameters $\xi_{1}$ and $\xi_{2}$ rather than $\Delta_{1}$ and $\Delta_{2}$. Grouping all $\Delta_{j}$ and $\xi_{j}$ into column vectors $\boldsymbol{\Delta}$ and $\boldsymbol{\xi}$, respectively, the general relationship between these quantities is
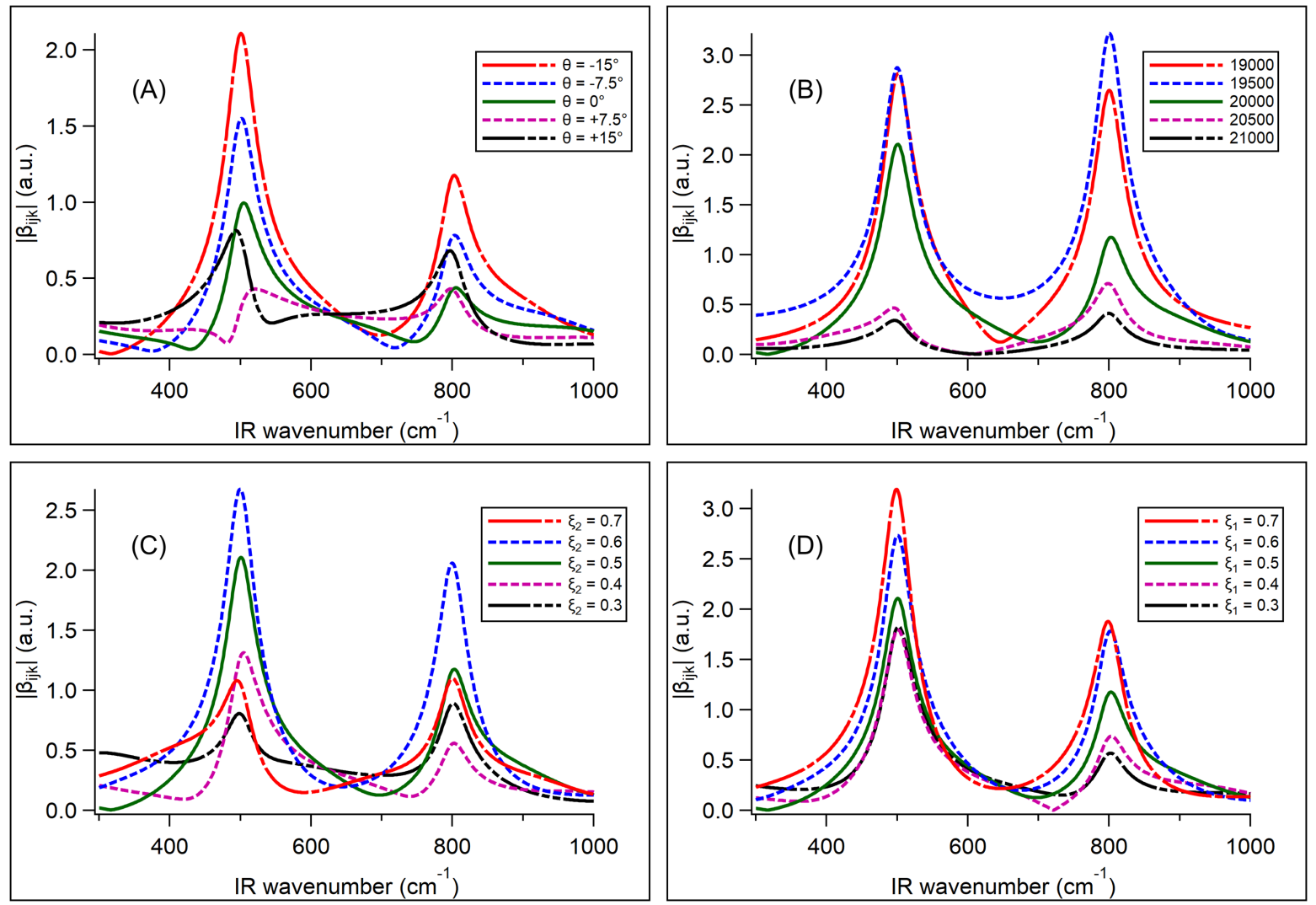

FIG. 5. Amplitudes of SFG hyperpolarizability $\left|\beta_{i j k}\right|$ as a function of the IR wavenumber for two modes experiencing mode mixing in the vibronic structure. Parameters are fixed as explained in the text and $A_{1}=10^{6}$ (a.u.). One parameter is varied in each panel: (A) mixing angle, (B) visible wavenumber $\left(\mathrm{cm}^{-1}\right)$, dimensionless displacements (C) $\xi_{2}$ and (D) $\xi_{1}$. 
$\boldsymbol{\Delta}=\sqrt{2 \hbar}\left(\boldsymbol{\omega}_{e}\right)^{-2} \mathbf{R}\left(\boldsymbol{\omega}_{g}\right)^{3 / 2} \boldsymbol{\xi}$, where $\mathbf{R}$ is the Duschinsky matrix, and $\boldsymbol{\omega}_{g}$ and $\boldsymbol{\omega}_{e}$ are the diagonal matrices of the vibration wavenumbers in the ground and excited states, respectively. Parameters $\xi_{j}$ are dimensionless, like $\bar{\Delta}_{j}$, but in the rotated basis for vibronic states (standard value $\xi_{1}=\xi_{2}=0.5$ ). In Fig. 5, we show the dispersion curves (using Eq. 6) for the amplitude of the molecular SFG hyperpolarizability, obtained by varying one parameter at a time while the others keep their standard value. Function $\Phi^{F S}(\omega)$ was calculated up to fourth order. We follow in this part the convention of Ref. 16 and plot SFG amplitudes rather than usual intensities for a direct comparison with their results.

We see that we perfectly reproduce the curves of Ref. 16, at a lower computational cost, validating our theoretical analysis of mode mixing. In particular, the complex structures in the lineshapes as a function of the mixing angle (Fig. 5A) are correctly accounted for. The main advantage of our procedure lies in the fact that we have to calculate the overlap spectral function only once, while we may vary at will the parameters not related to the distorted vibronic structure (mixing angles, visible and IR excitation wavelengths, amplitudes of each mode). In this sense, once the 0-0 energy $\left(\omega_{e g}^{0}\right)$, displacements $\left(\Delta_{j}\right)$ and distortion parameters $\left(\lambda_{j}\right)$ are known, this procedure allows curve fitting for the other unknown parameters.

We may comment on the choices for the simulations of Fig. 5. The authors in Ref. 16 have originally chosen to fix parameters $\xi_{1}$ and $\xi_{2}$ and transition energy $\omega_{e g}$. In our model, we have shown that the analysis must rather rely on the ground state displacements $\Delta_{j}$ and 0-0 transition energy $\omega_{e g}^{0}$. In fact, parameters $\xi_{j}$ relate to the rotated vibronic structure, whereas the IR beam excites vibration modes in the ground state. There is no direct relationship between the IR wavenumber and the tuned displacements $\xi_{1,2}$. In addition, contrary to $\Delta_{1}$ and $\Delta_{2}$, the variations of $\xi_{1,2}$ are not disconnected from those of $\theta$, so when the mixing angle is tuned, the amplitudes of the peaks and their relative phases also change. This explains why we dont see on panel (A) a clear evolution in amplitude from one peak to the other as a function of $\theta$, as expected. In addition, the transition energy $\omega_{e g}$ also depends on parameters $\Delta_{j}$ (or $\xi_{j}$ ), so tuning them has consequences on the actual position of the 0-0 line $\omega_{e g}^{0}$, which drives the resonance conditions. In concrete terms, for fixed $\xi_{1,2}$ values, the variations of $\omega_{e g}^{0}$ and $\Delta_{1,2}$ as a function of $\theta$ displace the relative positions of visible and SFG frequencies with respect to the electronic transition, the balance in amplitude between both peaks, and the depth of the electronic structure of each mode. For example, when $\theta$ is tuned from $-15^{\circ}$ 
to $+15^{\circ}, \omega_{e g}^{0}$ varies between 19604 and $19737 \mathrm{~cm}^{-1}$ (a reference value of $20000 \mathrm{~cm}^{-1}$ for the visible excitation therefore seems too high); $S_{1}$ between 0.47 and $0.04 ; S_{2}$ between 0.18 and 0.31, respectively. All these dependencies account for the complicated lineshapes observed in Fig. 5, which may not easily be qualitatively analyzed. The evolutions of the lineshapes in panels (A) and (C) would be much smoother by use of $\Delta_{j}$ and $\omega_{e g}^{0}$ parameters, to which the curves are very sensitive.
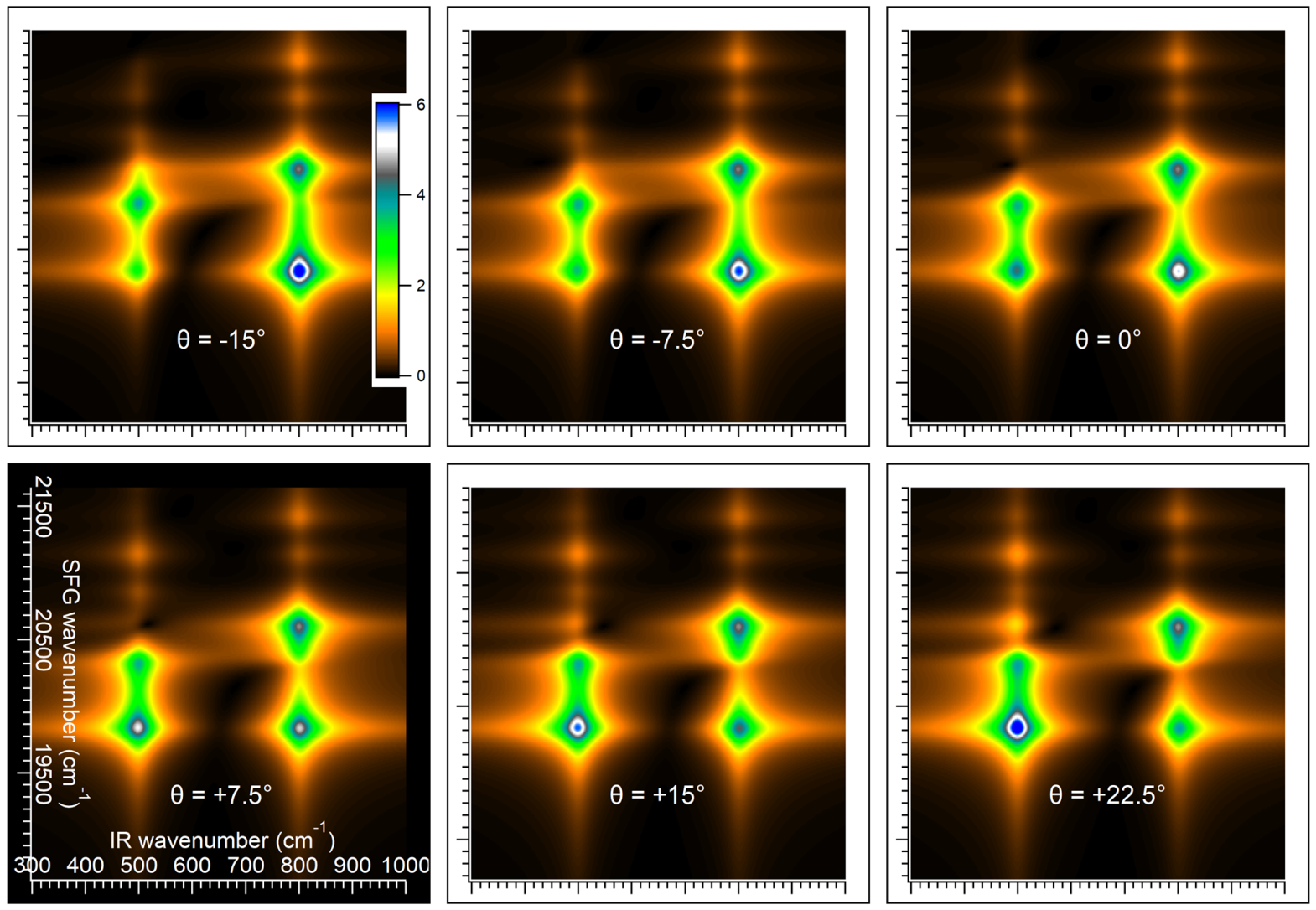

FIG. 6. Amplitudes of SFG hyperpolarizability $\left|\beta_{i j k}\right|$ for a two-mode system as a function of the IR and SFG wavenumbers, varying in the ranges $300-1000 \mathrm{~cm}^{-1}$, and $18700-21700 \mathrm{~cm}^{-1}$, respectively. Mode mixing angle is tuned between $-15^{\circ}$ and $+22.5^{\circ}$ as indicated in each panel. The parameters are described in the text, the main ones being $\omega_{e g}=20000 \mathrm{~cm}^{-1}, \omega_{1}^{g}=500 \mathrm{~cm}^{-1}, \omega_{2}^{g}=800 \mathrm{~cm}^{-1}$ and $\Delta_{1} / \sqrt{\hbar}=\Delta_{2} / \sqrt{\hbar}=0.02\left(\sqrt{\mathrm{cm}^{-1}}\right)^{-1}$.

In order to go further, we plot on Fig. 6 the evolutions of the same hyperpolarizability amplitudes as a function of the infrared and SFG wavenumbers for several values of the mixing angle $\theta$. Parameters have been chosen as above, except that we have fixed $\Delta_{1} / \sqrt{\hbar}=\Delta_{2} / \sqrt{\hbar}=0.02\left(\sqrt{\mathrm{cm}^{-1}}\right)^{-1}$. The two vibration modes are clearly seen on the spec- 
tra, and their amplitudes show two maxima in the visible range corresponding to the leading orders of the decomposition of Eq. 6. Here the excitation of higher vibronic states is small as a consequence of the rather small values chosen for $\Delta_{1,2}$. The first peak corresponds to the excitation of the electronic transition at the SFG frequency (this time, $\omega_{e g}^{0}$ is constant at $19826 \mathrm{~cm}^{-1}$ ), and the second one at the visible beam frequency, hence the alignment of the maxima on a horizontal and a tilted line, respectively. These two peaks also dominate the $D_{l}(\omega)$ curves in Part II B, and may be related to the two terms composing the SFG response in linear coupling case (Eq. 2). However, we clearly see that mode mixing has a strong impact on the excitation spectra. When $\theta$ is tuned from negative to positive values, the SFG intensity of the first peak is transferred from the high energy mode to the low energy one whereas the second peak remains stable. Higher order vibronic resonances also follow the amplitude trend of the first peak. Experimentally, the balance between peak intensities is predicted to be very sensitive to the mixing angle. We see that the evolution of the amplitudes of peaks 1 and 2 is not symmetric around $\theta=0^{\circ}$, the equilibrium point being located between $0^{\circ}$ and $+7.5^{\circ}$. This comes from the unequal amplitudes $A_{1}$ and $A_{2}$, which favor the second vibration mode. When comparing the situations for $-15^{\circ}$ and $+15^{\circ}$, we understand that we may not neglect mode mixing effects when analyzing experimental SFG spectra influenced by this phenomenon. Interpretation in the linear coupling frame will result in an incorrect balance between amplitudes $A_{1}$ and $A_{2}$, and consequently to erroneous conclusions on their infrared activities or vibronic displacement parameters.

In order to help understanding the difference between $\Delta$ and $\xi$ parameters, we compare in the Supplementary Material (Figures S6 to S8) analogous data for either constant $\Delta_{1,2}$ or constant $\xi_{1,2}$, plotted as a function of either the SFG or the visible wavenumber. In particular, in Figure S8, we illustrate the origins of the 1D curves on Fig. 5A and Fig. 5B. We also show that there is no clear trend for the evolution of the amplitudes as a function of $\theta$ when parameters $\xi_{1,2}$ are fixed instead of $\Delta_{1,2}$.

Another interest of our model for mode mixing lies in the fact that we may now separate the effects of the various terms in the excitation function (Eq. 6). Fig. 7 shows the decomposition of the total excitation spectrum $D_{1,2}^{\text {total }}(\omega)=D_{1,2}^{M I X}(\omega)+D_{1,2}^{C T}(\omega)$ for modes 1 and 2 into $D_{1,2}^{M I X}(\omega)$ and $D_{1,2}^{C T}(\omega)$ using the same parameters as in Fig. 6, for constant $\Delta_{1,2} / \sqrt{\hbar}$ as above, plotted as a function of mixing angle $\theta$ and wavenumber $\omega$. As shown in the Supplementary Material (Figure S9), terms $D_{1,2}^{\theta}$ appear negligible in front of the others (at least 


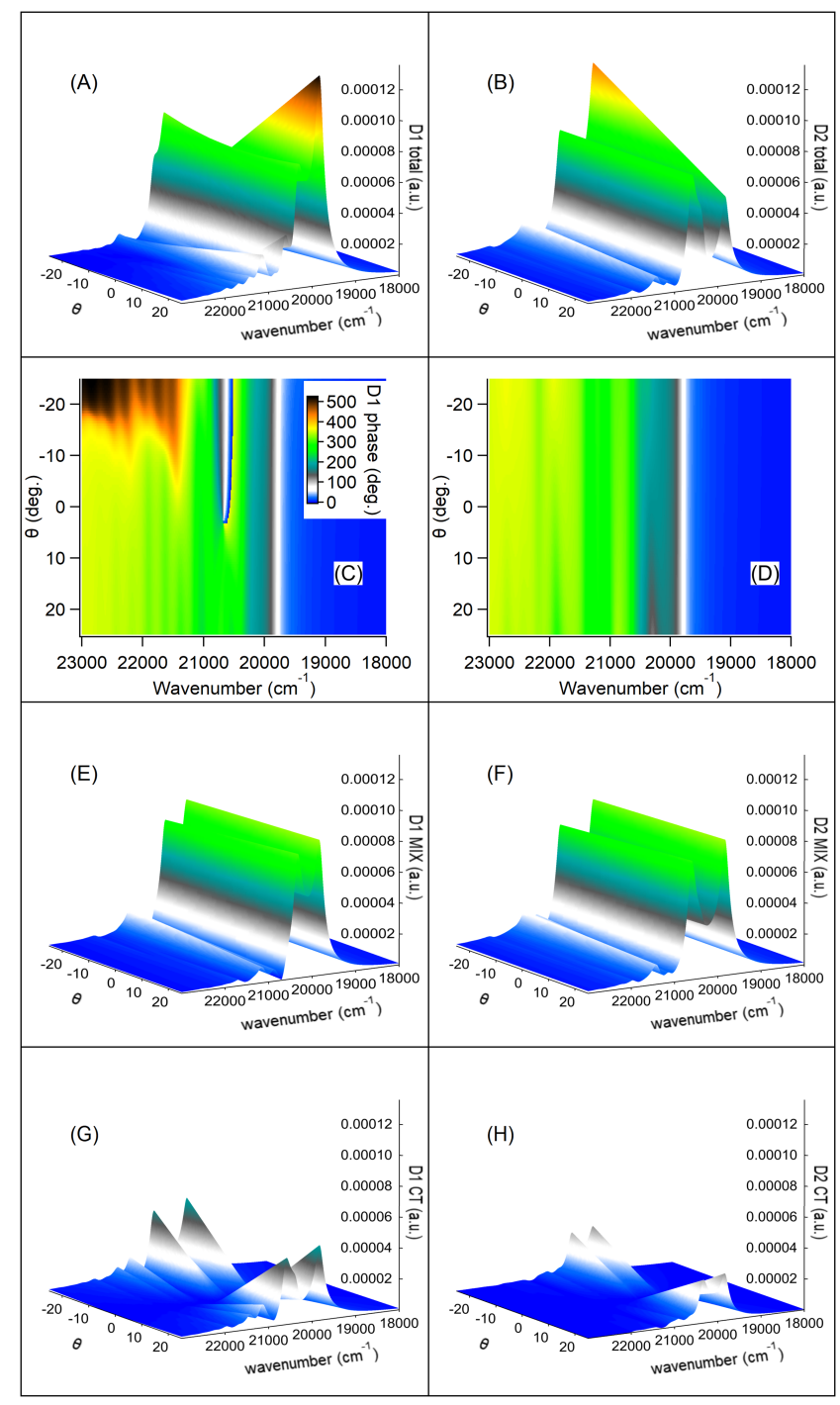

FIG. 7. Amplitudes and phases of the various terms of the excitation functions $D_{1}$ (left) and $D_{2}$ (right) when mode mixing is present, as a function of mixing angle (degrees) and wavenumber $\left(\mathrm{cm}^{-1}\right)$. Parameters are identical to Fig. 6. Total excitation functions; (A,B) amplitudes and (C,D) phases; (E,F) MIX terms only (amplitudes); (G,H) CT terms only (amplitudes). The six amplitude plots share a common scale.

30 times smaller than $D_{l}^{D I S}$ ), which may be anticipated as they involve a product of three $\Delta$ factors and therefore belong to the higher order terms. Consequently, $D_{l}^{M I X}(\omega)$ may be identified to $D_{l}^{D I S}(\omega)$ and remains essentially constant as a function of $\theta$ (Fig. 7E and 7F). The overall $\theta$-dependence therefore lies in $D_{1,2}^{C T}(\omega)$ only, proportionally to the mixing angle (Fig. $7 \mathrm{G}$ and $7 \mathrm{H}$ ), responsible for the $\theta$-dependent profiles shown in Fig. 6. Of course the 
phases of these terms (Fig. 7C and D) also contribute to the interference patterns between neighboring peaks on Fig. 6. For mode 2, and mode 1 in the positive range of mixing angles, the phase varies as a function of the wavelength but remains almost constant when the mixing angle is tuned. On the contrary, for mode 1 at negative $\theta$, above $20500 \mathrm{~cm}^{-1}$, the phase experiences rapid variations as a function of the wavenumber, and the $D_{1}$ sign for $\theta<0$ at high energies becomes opposite to the $\theta>0$ case. This comes from the fact that, in this region, $D_{1}^{C T}$ becomes slightly bigger in amplitude than $D_{1}^{M I X}$ and efficiently competes with it for the overall phase. In the particular case studied here, both modes share a common value for their coupling constants $\Delta_{1,2}$, and still the influence of mode mixing is obvious. However, when mixing involves two modes with very different values of $\Delta$, considering that $D_{1}^{M I X}$ is weighted by $\Delta_{1}$ and $D_{1}^{C T}$ by $\Delta_{2}$, we may expect the corrective term to become routinely bigger than the main $D^{M I X}$ (see below for an example). This illustrates that, even for moderate mode mixing, $D^{C T}$ terms may not be neglected, as they have a great influence both on amplitude and phase of the excitation functions. These results show that inclusion of mode mixing effects in the theoretical models, when they are present in the molecular vibronic structure, appears mandatory for an accurate experimental data analysis.

\section{Two modes in a multimode system: interference effects}

In order to assess the effects of distortions and mixing on a more realistic, but still model, example, we have chosen to investigate two nearby vibration modes of Rhodamine $6 \mathrm{G}$. We start again with the data from Ref. 5 and the same parameters as above for the electronic transition. This time, we use the whole calculated vibronic structure (theoretical frequencies and signed displacements of 31 vibronic modes) in order to calculate the $\Phi(\omega)$ function. As most publications dealing with the vibronic structure of R6G do not take into account mode distortion and mode mixing, ${ }^{6-11}$ we may play with these unknown parameters. Here we focus on mode mixing only and fix all $\lambda_{j}=1$, thus the overlap spectral function is simply $\Phi^{L C}(\omega)$. We first consider two modes, namely $\omega_{1}^{g}=1513 \mathrm{~cm}^{-1}, \omega_{2}^{g}=1652 \mathrm{~cm}^{-1}, \bar{\Delta}_{1}=-0.17$, $\bar{\Delta}_{2}=0.19, \Gamma_{1}^{g}=\Gamma_{2}^{g}=8 \mathrm{~cm}^{-1}$. We arbitrarily set their infrared activities $\partial \mu^{k} / \partial Q_{l}$ equal to simulate the DR-SFG intensities (calculated as $\left|\beta_{i j k}\right|^{2}$ ) for three mixing angles: $-15^{\circ}, 0^{\circ}$ and $+15^{\circ}$. In the Supplementary Material (Fig. S11), we see that the intensity spectra evolve as a function of the mixing angle in a way comparable to Fig. 6, except that, for a given 

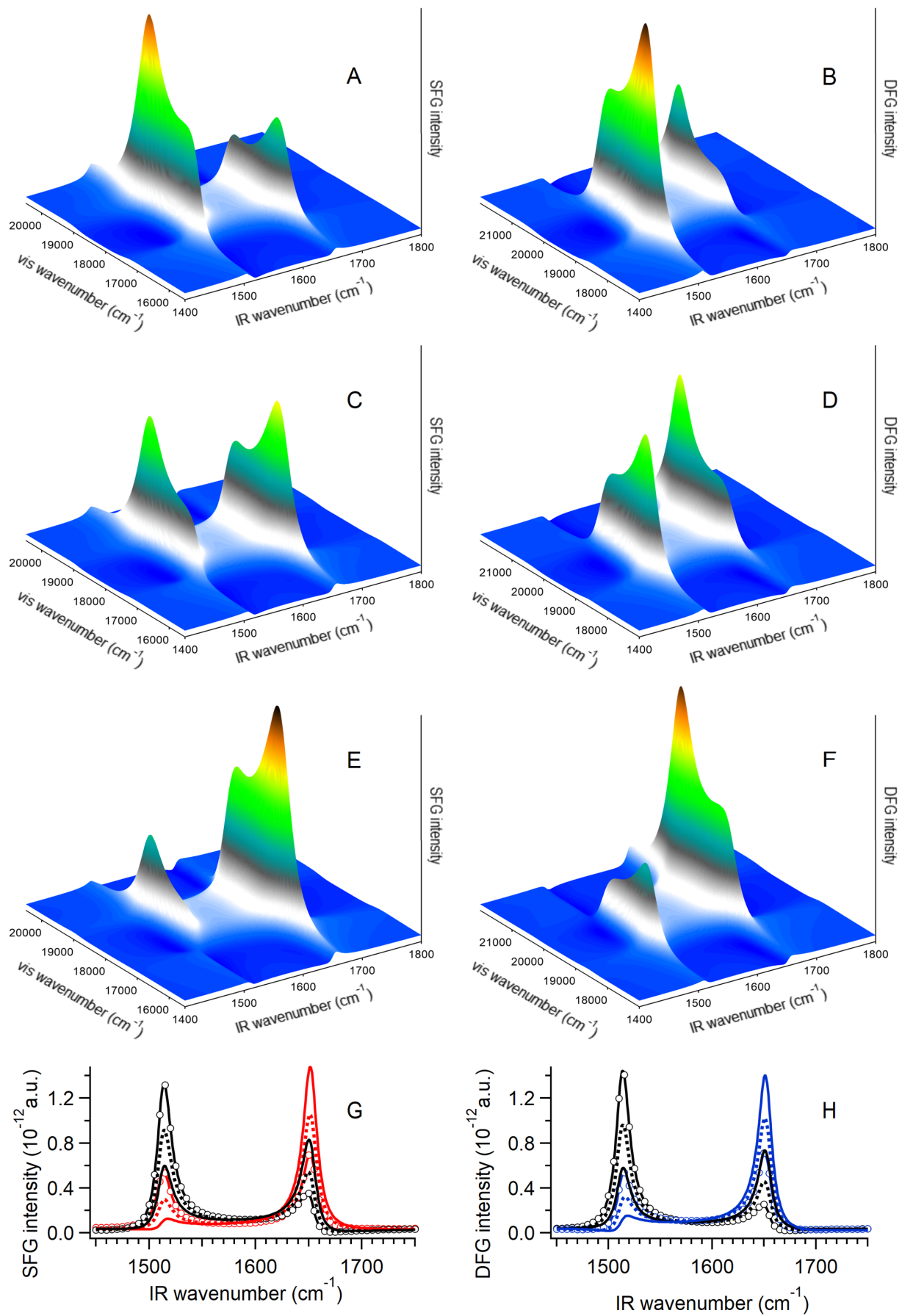

FIG. 8. DR-SFG (A,C,E,G) and DR-DFG (B,D,F,H) intensities from two vibration modes of R6G at 1513 and $1652 \mathrm{~cm}^{-1}$ as a function of the IR and visible wavenumbers, with $\mathrm{NR}=2 \times 10^{-7}$. Mode mixing angle is $-15^{\circ}(\mathrm{A}, \mathrm{B}), 0^{\circ}(\mathrm{C}, \mathrm{D})$ and $+15^{\circ}(\mathrm{E}, \mathrm{F})$. 1D IR spectra extracted at peak maxima (black for $\omega_{v i s} \approx \omega_{e g}^{0}$, red for SFG low energy peak, blue for DFG high energy peak) are shown in $(\mathrm{G}, \mathrm{H})$. Solid line: $+15^{\circ}$, dashed line: $0^{\circ}$, circles: $+15^{\circ}$. 
vibration mode, both peaks in the excitation spectrum share a common amplitude. This comes from the fact that the displacements shifts here take values lower than in Fig. 6, in fact more consistent with realistic systems. However, while both vibrations share an almost common absolute value for $\left(\partial \mu^{k} / \partial Q_{l}\right) \bar{\Delta}_{l}$, mode mixing still leads to a change in the intensity balance from one vibration to the other. In order to complete this picture with the phase effects, we suppose that the molecule is adsorbed onto a substrate giving birth to a nonvanishing SFG signal (e.g. gold, silicon) and draw the same intensity spectra while summing a constant small nonresonant (NR) term, with phase set to 0 , to the molecular $\beta_{i j k}$ (i.e. $\left|\beta_{i j k}+\mathrm{NR}\right|^{2}$ ). The comparison of the resulting spectra (Fig. 8) with the NRfree case (Fig. S11, same intensity scale) shows that the phases of both excitation peaks differ as their interference with the NR results in an asymetric change in intensity. Even if the NR intensity represents only $4 \%$ of the maximum intensity of the main peak, the effect on the spectra is dramatic: interference is either constructive (intensity increase) or destructive (intensity decrease) depending on the vibration mode and the resonance in the visible. When vibrational 1D spectra are extracted for the visible wavelengths corresponding to the resonant maxima (Fig. 8G), the intensity balance is tuned from one peak to the other in an asymmetric way.

It has been shown in the past that recording DFG spectra together with SFG may help analyzing the interference patterns. ${ }^{17,18}$ The equivalent DFG data are shown in Fig. 8 and Fig. S11. The resonances in the visible range show up in the DFG case for higher incoming visible energies than for $\mathrm{SFG}$, so that both techniques share a common maximum close to $\omega_{v i s}=\omega_{e g}^{0}$, with a second resonant peak at lower energy and higher energy for SFG and DFG, respectively. For their common resonance at $\omega_{v i s} \approx \omega_{e g}^{0}$, a direct comparison between Fig. $8 \mathrm{G}$ and $\mathrm{H}$ shows that they behave almost exactly in the same way as a function of $\theta$, even if the exact visible wavenumbers for the maxima vary from $18700 \mathrm{~cm}^{-1}$ for $\mathrm{SFG}$ to $18900 \mathrm{~cm}^{-1}$ for DFG. For a fixed mixing angle, intensity balance is reversed between SFG and DFG as far as the extremal peaks in the visible range (i.e. high energy for SFG, low energy for DFG) are concerned. These results show that an overall SFG/DFG symmetry may be recovered for the central peak and even for the extremal ones, but in the latter case we must keep in mind that experimental spectra must be recorded at two distinct visible wavenumbers, around 574 and $491 \mathrm{~nm}$ for SFG and DFG, respectively.

For a direct comparison with experiments, a series of vibrational spectra (i.e. as a func- 
tion of the IR wavenumber) will usually be sequentially recorded while tuning the visible wavenumber. For data analysis, the easiest procedure is to perform a Lorentzian fit of the spectra as a function of the IR wavenumber, the numerator being a complex function of the visible and SFG wavenumbers. ${ }^{12}$ In a practical way, many authors simplify the analysis by fitting each IR SFG spectrum using Lorentzian functions with a constant complex numera-

tor, then study the variation of this constant with the visible or SFG wavenumber. ${ }^{6,19-21}$. As this approximation is strictly speaking not enforced (i.e. the numerator of the Lorentzian also varies with the IR wavenumber), we have checked whether this procedure is acceptable in the present case. We have considered the twelve spectra in Fig. 8G and $\mathrm{H}$, and compared the parameters obtained by curve fitting to the exact values of the excitation functions at peak maximum. The values of the Lorentzian numerators obtained by both methods slightly differ but the phase accuracy remains within $2^{\circ}$ and the absolute amplitudes deviate by $4 \%$ at maximum. Data fitting also leads to errors in the NR amplitude, up to $4 \%$. The errors grow up to $6^{\circ}$ in phase and $4 \%$ in amplitude, with a NR deviating by more than $10 \%$ when the same analysis is performed with two other vibration modes, closer to each other $\left(14 \mathrm{~cm}^{-1}\right)$. More important, the phase difference between the two vibration modes differs from zero, and reaches values up to $23^{\circ}$. As a consequence, the complex Lorentzians require numerators with distinct phases (this issue is sometimes not addressed in the literature ${ }^{6}$ ), which raises additional difficulties: as expected, we have found four distinct sets of parameters for every single spectrum while performing curve fitting. ${ }^{22}$ Considering the variability of the experimental peak intensities (Fig. 7A and B) and phases (Fig. 7C and D) with the mixing angle, it is impossible to know which set corresponds to the correct parameters. This constitutes a major issue as for data interpretation of DR-SFG and DR-DFG spectra.

\section{E. Realistic vibronic structure model}

In order to illustrate the importance of mode distortion and mode mixing in practical DR-SFG experiments, we extend the previous analysis to the full vibronic structure of rhodamine 6G. We have calculated by DFT (see Supplementary Material for details) the vibrational structures of the ground and first excited electronic states of the molecule after geometry optimisation. Comparison between both vibrational structures allows to extract the displacement vector and mode mixing matrix. In a second step, we have reduced the 


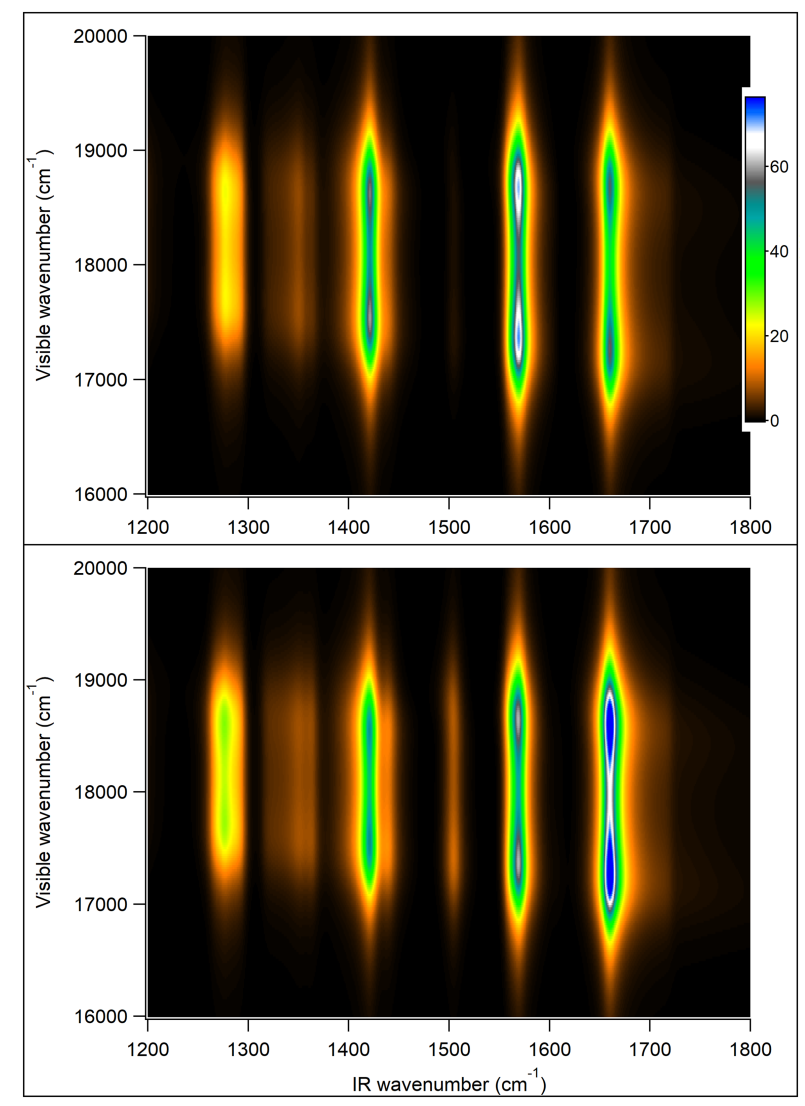

FIG. 9. Simulated DR-SFG intensities $\left|\beta_{i j k}\right|^{2}$ of model rhodamine $6 \mathrm{G}$ as a function of visible and IR wavenumbers $\left(\mathrm{cm}^{-1}\right)$, with mode distortion and mode mixing excluded (top) and included (bottom).

Duschinsky matrix to a block diagonal 2x2 matrix. Using Eq. 6, it becomes possible to calculate the DR-SFG spectrum of rhodamine $6 \mathrm{G}$ as a function of incoming visible and IR frequencies, at the Condon level of approximation, in terms of molecular hyperpolarizability components. A comparison to experimental spectra is then possible on condition that the orientation of the molecule on the surface is known, allowing projection of SFG, visible and IR dipole moments from the molecular to the laboratory frame. ${ }^{23}$ Even for a fixed set of beam polarizations (e.g. ssp), the visible and SFG electronic transition dipole moments, and the wavelength dispersion in the visible, factorize when comparing vibration modes with one another (see part I), but the excitation of the IR dipole moments $\partial \mu^{k} / \partial Q_{l}$ by light still depends on the molecular orientation. As our goal is not to compare our simulations with experimental spectra, we do not consider a particular molecular orientation ${ }^{11}$ and plot an averaged $\left|\beta_{i j k}\right|^{2}$ intensity, weighting each vibration mode $l$ by $\left\langle\partial \mu / \partial Q_{l}\right\rangle$ corresponding to 
its IR activity averaged over all orientations. All vibrations share a common $\Gamma_{l}^{g}=8 \mathrm{~cm}^{-1}$.

In Fig. 9 we compare the SFG spectra as a function of the visible and IR incoming wavenumbers calculated with and without account of mode mixing and mode distortions. Parameters of the electronic resonance are taken as before as $\omega_{e g}^{0}=18800 \mathrm{~cm}^{-1}$ and $\Gamma_{e g}=$ $400 \mathrm{~cm}^{-1}$. In the $1200-1800 \mathrm{~cm}^{-1}$ IR range, six peaks mainly appear on the spectrum, some of them broadened by overlapping neighbouring modes. The two main prominent features lie at 1660 and $1569 \mathrm{~cm}^{-1}$, in line with other publications on the same molecule. . $^{7,8,10,11}$ We note that the Herzberg-Teller (HT) active modes do not appear on our calculation, restricted to Franck-Condon active modes. ${ }^{11}$ However, the mode at $1504 \mathrm{~cm}^{-1}$, which is known to be only HT-active ${ }^{11}$, is absent indeed on the top spectrum as expected, but becomes visible when quadratic electron-vibration coupling is allowed. It appears that, even if this mode is IR-active but only weakly Franck-Condon active, it borrows its intensity by mode mixing (with positive angle) from the neighbouring $1569 \mathrm{~cm}^{-1}$ mode. Conversely, the latter shows a decreasing intensity for the same reason. As a consequence, the balance in intensity between the 1569 and $1660 \mathrm{~cm}^{-1}$ peaks is reversed when mode mixing is taken into account, all the more that the latter also borrows some intensity from a very weakly IRactive mode at $1608 \mathrm{~cm}^{-1}$, with a negative coupling angle. For these particular peaks, and to a lower extent for all vibration modes visible on Fig. 9, we see that quadratic coupling effect changes the simulated vibrational and electronic lineshapes, which has a strong impact on the parameters extracted from the analysis of experimental data (e.g. coupling parameters $\Delta_{l}$ and IR activities $\left.\partial \mu^{k} / \partial Q_{l}\right)$.

This is illustrated in Fig. 10A, where the spectra with and without quadratic coupling effects are superimposed for $\omega_{v i s}=18600 \mathrm{~cm}^{-1}$. In addition to the features described above, the intensities of peaks at 1196, 1276, 1361 and the high energy shoulder at $1439 \mathrm{~cm}^{-1}$ are also modified by mode distortion and mode mixing. The choice of this specific visible frequency is explained in Fig. 10B, where the amplitudes of several vibration modes spanning the IR range (prominent or not in the SFG spectrum) are displayed as a function of the visible wavenumber and normalized to 1 . We see that, as a consequence of interference between the two peaks, the effective maximum for the high energy resonance is redshifted from the theoretical $\omega_{v i s}=\omega_{e g}^{0}$ towards an average value of $18600 \mathrm{~cm}^{-1}$. In addition, the peak intensities show the expected behaviour as a function of the IR wavenumber (Fig. 4), i.e. the two distinct maxima overlap at high IR energy (with a valley of moderate depth in 


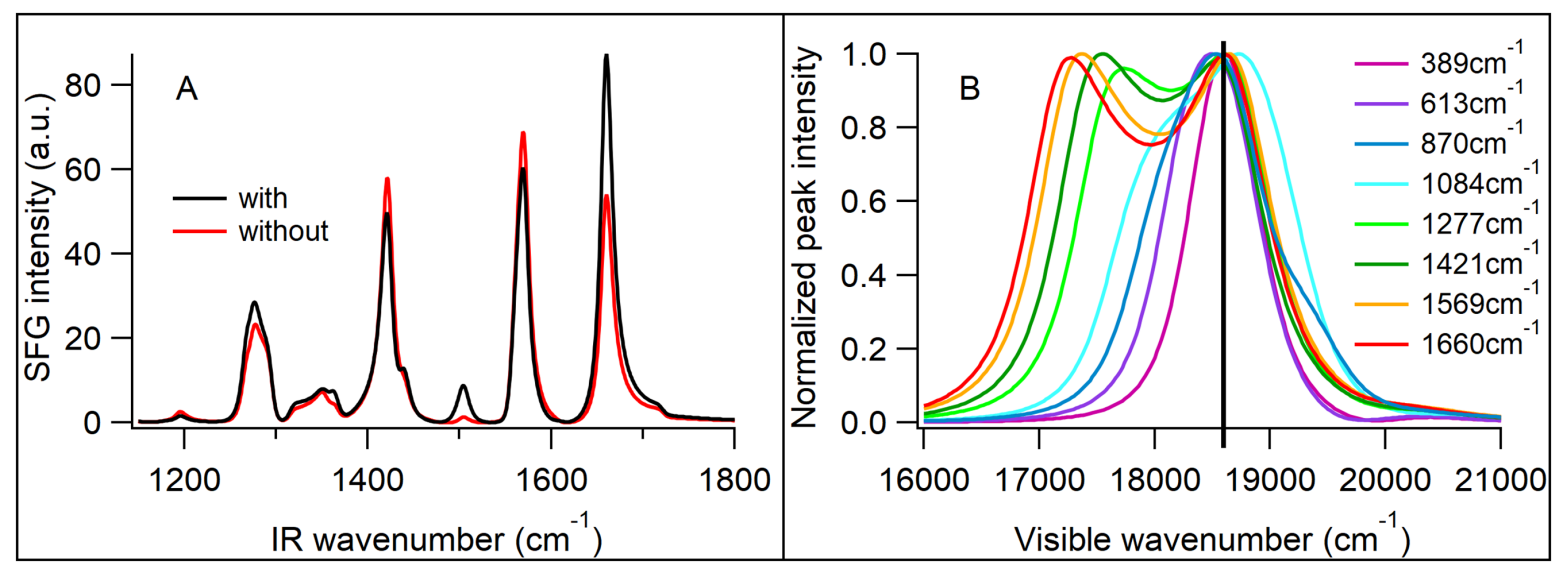

FIG. 10. A: comparison of the SFG intensities $\left|\beta_{i j k}\right|^{2}$ of model rhodamine $6 \mathrm{G}$ extracted from Fig. 9 at a visible wavenumber of $18600 \mathrm{~cm}^{-1}$ calculated with and without including mode mixing and mode distortions. B: peak intensities of several vibration modes as a function of the visible wavenumber, normalized to unity to ease comparison. Vertical line is at $18600 \mathrm{~cm}^{-1}$.

between) and merge into a broader peak at low IR energy.

\section{CONCLUSION}

Doubly resonant SFG and DFG spectroscopies require a specific formalism for their analysis, which essentially differs from the singly vibrational resonant case. For every vibration mode, the classical IR complex Lorentzian formulation for vibrational resonances holds but its numerator (the excitation spectrum of the mode) involves several wavenumber-dependent factors related to the molecular vibronic structure. For low amplitude mode mixing limited to couples of vibration modes, these factors are elegantly recast into the spectral overlap function $\Phi(\omega)$, which only depends on mode distortion through frequency shifts. The $\Phi$ function may be extracted from experimental absorption data, or calculated through a sum-overstates algorithm. The depth of this calculation depends on the amplitude of displacements along normal mode coordinates of all the vibration modes in the vibronic structure.

As compared to the linear coupling case, i.e. without account of mode mixing and mode distortions, inclusion of mode distortions mainly modifies the resonance conditions through vibrational frequency shifts in the excited state. This phenomenon is completely taken care of in the $\Phi(\omega)$ by changing $\Phi^{L C}$ into $\Phi^{F S}$. In addition, for high values of the displacements 
along normal modes, the influence of higher order terms (i.e. involving harmonics and overtones of the vibrations) increases with the distortion. Including mode mixing leads to additional angle-dependent terms in the excitation spectra. The effects are more spectacular than with mode distortions: SFG intensity is deeply balanced from one mode to the other when the mixing angle varies on either side of zero, preventing from a direct interpretation of the absolute peak intensity in terms of infrared transition dipole moment and normal mode displacement alone. When the mixed modes have very unbalanced amplitudes (e.g. one is very IR active and the other one completely or almost IR-inactive), intensity transfer form the IR-active mode may make the IR-inactive mode look IR-active. Finally, the phases of the vibration modes vary under the influence of mode mixing, creating new interference patterns with the neighbouring modes and with a non-resonant contribution from the substrate, when present. This impacts again on the apparent intensity of each peak, but also makes fitting procedure with Lorentzians more difficult due to the existence of several equivalent sets of parameters.

The results presented here show that quadratic electron-vibration couplings, responsible for mode distortions and mode mixing, must be taken into account in order to correctly estimate the vibrational amplitudes from DR-SFG and DR-DFG experiments, then recover the molecular parameters like vibrational transition dipole moments and vibronic coupling constants. We expect in the future some improvements on the present theory and its practical implementation, for example by building on the full vibronic theory ${ }^{16}$ in order to avoid excessively simplifying the Duschinsky matrix, or by inserting quadratic effects into the Herzberg-Teller formalism, ${ }^{11}$ which generates a second family of active modes. We may also expect that a generalized use of computational chemistry allows a better access to realistic molecular vibronic structures, in order to implement normal mode displacements, distortion parameters and Duschinsky matrices compatible with real molecules into data analysis of doubly resonant second order nonlinear optical spectroscopies.

\section{SUPPLEMENTARY MATERIAL}

See Supplementary Material for additional figures on the calculation of the overlap spec-

tral function and on the effects of mode distortions and mode mixing on excitation profiles and SFG/DFG spectra, and for details on the calculation of the vibronic structure of rho- 
damine $6 \mathrm{G}$.

\section{ACKNOWLEDGMENTS}

We thank Dr. Abderrahmane Tadjeddine and Dr. Thomas Noblet for their fruitful comments on this work and their suggestions of improvements on this manuscript.

\section{DATA AVAILABILITY}

The data that supports the findings of this study are available within the article and its supplementary material.

\section{Appendix A}

The excitation functions introduced in Eq. 6 have the following expressions:

$$
D_{l}^{M I X}(\omega)=D_{l}^{D I S}(\omega)+D_{l}^{\theta}(\omega)
$$

where $D_{l}^{D I S}$ refers to Eq. 4 and

$$
\begin{gathered}
D_{l}^{\theta}(\omega)=-\frac{\Delta_{l} \Delta_{1} \Delta_{2} \theta\left(\omega_{1}^{g}-\omega_{2}^{g}\right)}{4 \hbar \quad}\left[\Phi^{F S}(\omega)-\Phi^{F S}\left(\omega-\omega_{l}^{e}\right)\right. \\
-\Phi^{F S}\left(\omega-\omega_{1}^{e}\right)+\Phi^{F S}\left(\omega-\omega_{l}^{e}-\omega_{1}^{e}\right) \\
-\Phi^{F S}\left(\omega-\omega_{2}^{e}\right)+\Phi^{F S}\left(\omega-\omega_{l}^{e}-\omega_{2}^{e}\right) \\
\left.+\Phi^{F S}\left(\omega-\omega_{1}^{e}-\omega_{2}^{e}\right)-\Phi^{F S}\left(\omega-\omega_{l}^{e}-\omega_{1}^{e}-\omega_{2}^{e}\right)\right] \\
\begin{array}{c}
D_{1}^{C T}(\omega)=+\frac{\Delta_{2} \theta}{4 \omega_{1}^{g}}\left\{\left(\omega_{1}^{g}+\omega_{2}^{g}\right)\left[\Phi^{F S}(\omega)-\Phi^{F S}\left(\omega-\omega_{2}^{e}\right)\right]\right. \\
\left.-\left(\omega_{1}^{g}-\omega_{2}^{g}\right)\left[\Phi^{F S}\left(\omega-\omega_{1}^{e}\right)-\Phi^{F S}\left(\omega-\omega_{1}^{e}-\omega_{2}^{e}\right)\right]\right\} \\
D_{2}^{C T}(\omega)=-\frac{\Delta_{1} \theta}{4 \omega_{2}^{g}}\left\{\left(\omega_{1}^{g}+\omega_{2}^{g}\right)\left[\Phi^{F S}(\omega)-\Phi^{F S}\left(\omega-\omega_{1}^{e}\right)\right]\right. \\
\left.-\left(\omega_{2}^{g}-\omega_{1}^{g}\right)\left[\Phi^{F S}\left(\omega-\omega_{2}^{e}\right)-\Phi^{F S}\left(\omega-\omega_{1}^{e}-\omega_{2}^{e}\right)\right]\right\}
\end{array}
\end{gathered}
$$


For the general case of $\mathrm{P}$ modes involved in mode mixing by pairs, for each $(\mathrm{i}, \mathrm{j})$ pair corresponds an angle $\theta_{i j}$, and the excitation functions involved in the SFG hyperpolarizability (Eq. 7) become

$$
\begin{gathered}
D_{l}^{\theta}(\omega)=-\Delta_{l} \sum_{(i, j)=(1,2)}^{(P-1, P)} \frac{\Delta_{i} \Delta_{j} \theta_{i j}\left(\omega_{i}^{g}-\omega_{j}^{g}\right)}{4 \hbar}\left[\Phi^{F S}(\omega)-\Phi^{F S}\left(\omega-\omega_{l}^{e}\right)\right. \\
-\Phi^{F S}\left(\omega-\omega_{i}^{e}\right)+\Phi^{F S}\left(\omega-\omega_{l}^{e}-\omega_{i}^{e}\right) \\
-\Phi^{F S}\left(\omega-\omega_{j}^{e}\right)+\Phi^{F S}\left(\omega-\omega_{l}^{e}-\omega_{j}^{e}\right) \\
\left.+\Phi^{F S}\left(\omega-\omega_{i}^{e}-\omega_{j}^{e}\right)-\Phi^{F S}\left(\omega-\omega_{l}^{e}-\omega_{i}^{e}-\omega_{j}^{e}\right)\right], \\
D_{i}^{C T}(\omega)=+\frac{\Delta_{j} \theta_{i j}}{4 \omega_{i}^{g}\left\{\left(\omega_{i}^{g}+\omega_{j}^{g}\right)\left[\Phi^{F S}(\omega)-\Phi^{F S}\left(\omega-\omega_{j}^{e}\right)\right]\right.} \\
\left.-\left(\omega_{i}^{g}-\omega_{j}^{g}\right)\left[\Phi^{F S}\left(\omega-\omega_{i}^{e}\right)-\Phi^{F S}\left(\omega-\omega_{i}^{e}-\omega_{j}^{e}\right)\right]\right\},
\end{gathered}
$$

and

$$
\begin{aligned}
D_{j}^{C T}(\omega)=-\frac{\Delta_{i} \theta_{i j}}{4 \omega_{j}^{g}}\left\{\left(\omega_{i}^{g}+\omega_{j}^{g}\right)[\right. & \left.\Phi^{F S}(\omega)-\Phi^{F S}\left(\omega-\omega_{i}^{e}\right)\right] \\
& \left.-\left(\omega_{j}^{g}-\omega_{i}^{g}\right)\left[\Phi^{F S}\left(\omega-\omega_{j}^{e}\right)-\Phi^{F S}\left(\omega-\omega_{i}^{e}-\omega_{j}^{e}\right)\right]\right\}
\end{aligned}
$$

for the first and second mode of the (i,j) pair, respectively.

\section{REFERENCES}

${ }^{1}$ B. Busson, J. Chem. Phys. (2020).

${ }^{2}$ M. Hayashi, S. H. Lin, M. B. Raschke, and Y. R. Shen, J. Phys. Chem. A 106, 2271 (2002).

${ }^{3}$ X. Zhuang, P. B. Miranda, D. Kim, and Y. R. Shen, Phys. Rev. B 59, 12632 (1999).

${ }^{4}$ F. Santoro, A. Lami, R. Improta, and V. Barone, J. Chem. Phys. 126, 184102 (2007).

${ }^{5}$ J. Guthmuller and B. Champagne, ChemPhysChem 9, 1667 (2008).

${ }^{6}$ M. Raschke, M. Hayashi, S. Lin, and Y. Shen, Chem. Phys. Lett. 359, 367 (2002).

${ }^{7}$ J. Guthmuller and B. Champagne, J. Phys. Chem. A 112, 3215 (2008).

${ }^{8}$ S. Shim, C. M. Stuart, and R. A. Mathies, ChemPhysChem 9, 697 (2008).

${ }^{9}$ C. B. Milojevich, D. W. Silverstein, L. Jensen, and J. P. Camden, J. Am. Chem. Soc. 133, 14590 (2011). 
${ }^{10}$ C. B. Milojevich, D. W. Silverstein, L. Jensen, and J. P. Camden, J. Phys. Chem. C 117, $3046(2013)$.

${ }^{11}$ P. A. Weiss, D. W. Silverstein, and L. Jensen, J. Phys. Chem. Lett. 5, 329 (2014).

${ }^{12}$ S. Sengupta, L. Bromley III, and L. Velarde, J. Phys. Chem. C 121, 3424 (2017).

${ }^{13}$ T. Noblet, L. Dreesen, S. Boujday, C. Méthivier, B. Busson, A. Tadjeddine, and C. Humbert, Commun. Chem. 1, 76 (2018).

${ }^{14}$ L. Dalstein, C. Humbert, M. Ben Haddada, S. Boujday, G. Barbillon, and B. Busson, J. Phys. Chem. Lett. 10, 7706 (2019).

${ }^{15}$ B. Busson and L. Dalstein, J. Phys. Chem. C 123, 26597 (2019).

${ }^{16}$ J. C. Vallet, A. J. Boeglin, J. P. Lavoine, and A. A. Villaeys, Phys. Rev. A 53, 4508 (1996).

${ }^{17}$ J. Y. Huang and Y. R. Shen, Phys. Rev. A 49, 3973 (1994).

${ }^{18}$ A. Le Rille and A. Tadjeddine, J. Electroanal. Chem. 467, 238 (1999).

${ }^{19}$ T. Miyamae, Y. Miyata, and H. Kataura, J. Phys. Chem. C 113, 15314 (2009).

${ }^{20}$ T. Nagahara, K. Kisoda, H. Harima, M. Aida, and T. A. Ishibashi, J. Phys. Chem. B 113, 5098 (2009).

${ }^{21}$ M. Raab, J. C. Becca, J. Heo, C.-K. Lim, A. Baev, L. Jensen, P. N. Prasad, and L. Velarde, J. Chem. Phys. 150, 114704 (2019).

${ }^{22}$ B. Busson and A. Tadjeddine, J. Phys. Chem. C 113, 21895 (2009).

${ }^{23}$ S. Roy, K.-K. Hung, U. Stege, and D. K. Hore, Appl. Spectrosc. Rev. 49, 233 (2014). 